\title{
SYNTHESIS AND ANTIBACTERIAL ACTIVITY OF NEW SULPHONAMIDES OF PYRIMIDINE
}

Jerzy Cieplik ${ }^{2}$, Janusz Pluta ${ }^{\text {b }}$, Olaf Gubrynowicz ${ }^{\text {b }}$

a) Department of Organic Chemistry, Medical Academy, Grodzka 9, 50-137 Wrocław, Poland

b) Department of Applied Pharmacy, Medical Academy, Szewska 38, 50-137 Wroclaw, Poland

\section{Summary}

The paper presents the synthesis of 5-substituted pyrimidine sulphonamides as well as the results of studies on the aritibacterial and antifungal activity of obtained derivatives.

\section{Introduction}

Results of previous research on synthesis and biological properties of pyrimidine ring have demonstrated that the system is capable of extremely potent biological activity. The obtained derivatives revealed both cytostatic [1,2], immunomodulating [3,4] and first of all antibacterial $[5,6,7]$ properties. That is why we found useful to carry out a number of syntheses aiming at obtaining 5 -substituted pyrimidine sulphonamides.

\section{CHEMISTRY}

The substrate in our studies was 4-aryl-6-methyl-2-phenyl-5-hydroxymethylpyrimidine (1), which after treatment with $\mathrm{SOCl}_{2}$, was transformed into 2-aryl-6-methyl-2-phenyl-5chloromethylpyrimidine (2). Next 5-substituted pyrimidine sulphonamides were obtained by means of two methods. The first one consisted in treatment of 5-chlorderivative of pyrimidine 2 with aqueous solution of ammonia gave 5-aminoderivative 3 , which was condensed with p-toluilosulphonic acid chloride giving adequate sulphonamides. The second method consisted in direct action of $\mathrm{N}$ acetylsulfanilamide on 5-chloroderivative of pyrimidine 2 . Despite of several trials, we failed to obtain double sulphonamides, compound 5 . The arylaminegroup at site 4 of the pyrimidine ring does not react with sulphonic chlorides.

Physical and spectral properties of the compounds are given in Table 1.

Key words Pyrimidine, sulphonamides, antibacterial activity

(*) Herrn Prof. Fleischhacker zum 65. Geburtstag gewidmet 
<smiles>[R]c1ccc(Nc2nc(-c3ccccc3)nc(C)c2CO)cc1</smiles><smiles>CI</smiles><smiles>[R]1ccc2ccccc12</smiles><smiles>CCCNc1nc(-c2ccccc2)nc(C)c1CCl</smiles>
2<smiles>CC</smiles><smiles>[R]c1ccc(Nc2nc(-c3ccccc3)nc(C)c2CN[SeH])cc1</smiles><smiles>[R]Cc1ccc(N(c2ccc(C)cc2)c2nc(-c3ccccc3)nc(C)c2CNS(=O)(=O)c2ccc(C)cc2)cc1</smiles><smiles>CC(C)(C)[V]</smiles><smiles>Cc1nc(-c2ccccc2)nc(N)c1CN</smiles><smiles>[R]c1cccc(N)c1</smiles><smiles>Cc1ccc(O[SH](=O)(NCc2c(C)nc(-c3ccccc3)nc2N)c2ccccc2)cc1</smiles><smiles>[R]c1ccccc1Nc1nc(-c2ccccc2)nc(C)c1CNS(=O)(=O)c1ccc(N)cc1</smiles>

Scheme 1 
Physical and spectral of the compounds are given in Table 1.<smiles>[R]NCc1c(C)nc(-c2ccccc2)nc1Nc1ccccc1</smiles>

\begin{tabular}{|c|c|c|c|c|c|c|c|c|}
\hline $\begin{array}{l}\text { Co } \\
\mathrm{mp}\end{array}$ & $\mathrm{R}$ & $\mathrm{R}^{\prime}$ & Formula & M.P. & Yield & A N A & $\begin{array}{l}\mathrm{L} Y \\
\text { calc. } \\
\text { found }\end{array}$ & S IS \\
\hline & & & (M.W.) & $\left({ }^{\circ} \mathrm{C}\right)$ & $(\%)$ & $\mathrm{C}$ & $\mathrm{H}$ & $\mathrm{N}$ \\
\hline $3 \mathbf{a}$ & $\mathrm{H}$ & $\mathrm{H}$ & $\begin{array}{l}\mathrm{C}_{18} \mathrm{H}_{18} \mathrm{~N}_{4} \\
(290)\end{array}$ & $117-119$ & 92.3 & $\begin{array}{l}74.48 \\
74.56 \\
\end{array}$ & $\begin{array}{l}6.20 \\
6.36 \\
\end{array}$ & $\begin{array}{l}19.31 \\
19.52 \\
\end{array}$ \\
\hline $3 \mathrm{~b}$ & $4-\mathrm{Cl}$ & $\mathrm{H}$ & $\begin{array}{l}\mathrm{C}_{18} \mathrm{H}_{17} \mathrm{~N}_{4} \mathrm{Cl} \\
(324)\end{array}$ & $148-150$ & 88.2 & $\begin{array}{l}66.66 \\
66.50 \\
\end{array}$ & $\begin{array}{l}5.24 \\
4.95 \\
\end{array}$ & $\begin{array}{l}17.28 \\
16.96 \\
\end{array}$ \\
\hline $3 \mathrm{c}$ & $4-\mathrm{OC}_{2} \mathrm{H}_{5}$ & $\mathrm{H}$ & $\begin{array}{l}\mathrm{C}_{20} \mathrm{H}_{22} \mathrm{~N}_{4} \mathrm{O} \\
(334)\end{array}$ & $109-111$ & 85.3 & $\begin{array}{l}71.85 \\
71.55 \\
\end{array}$ & $\begin{array}{l}6.58 \\
6.50 \\
\end{array}$ & $\begin{array}{l}16.76 \\
17.03 \\
\end{array}$ \\
\hline $3 \mathrm{~d}$ & $4-\mathrm{OH}$ & $\mathrm{H}$ & $\begin{array}{l}\mathrm{C}_{18} \mathrm{H}_{18} \mathrm{~N}_{4} \mathrm{O} \\
(306)\end{array}$ & $110-112$ & 82.2 & $\begin{array}{l}70.58 \\
70.62 \\
\end{array}$ & $\begin{array}{l}5.88 \\
5.90 \\
\end{array}$ & $\begin{array}{l}18.30 \\
18.26 \\
\end{array}$ \\
\hline $3 \mathrm{e}$ & $4-\mathrm{CH}_{3}$ & $\mathrm{H}$ & $\begin{array}{l}\mathrm{C}_{19} \mathrm{H}_{20} \mathrm{~N}_{4} \\
(304)\end{array}$ & $120-122$ & 79.2 & $\begin{array}{l}75.00 \\
75.22 \\
\end{array}$ & $\begin{array}{l}6.57 \\
6.66 \\
\end{array}$ & $\begin{array}{l}18.42 \\
18.56 \\
\end{array}$ \\
\hline $4 a$ & $\mathrm{H}$ & $-\mathrm{SO}_{2}-\mathrm{C}_{6} \mathrm{H}_{4}-4-\mathrm{CH}_{3}$ & $\begin{array}{l}\mathrm{C}_{25} \mathrm{H}_{24} \mathrm{~N}_{4} \mathrm{O}_{2} \mathrm{~S} \\
(444)\end{array}$ & $164-166$ & 64.5 & $\begin{array}{l}67.58 \\
67.40 \\
\end{array}$ & $\begin{array}{l}5.40 \\
5.65 \\
\end{array}$ & $\begin{array}{l}12.61 \\
12.33 \\
\end{array}$ \\
\hline $4 \mathrm{~b}$ & $4-\mathrm{Cl}$ & $-\mathrm{SO}_{2}-\mathrm{C}_{6} \mathrm{H}_{4}-4-\mathrm{CH}_{3}$ & $\begin{array}{l}\mathrm{C}_{25} \mathrm{H}_{23} \mathrm{~N}_{4} \mathrm{O}_{2} \mathrm{SCl} \\
(478)\end{array}$ & $138-140$ & 63.5 & $\begin{array}{l}62.76 \\
62.54 \\
\end{array}$ & $\begin{array}{l}4.81 \\
5.12 \\
\end{array}$ & $\begin{array}{l}11.47 \\
11.62 \\
\end{array}$ \\
\hline $4 c$ & $4-\mathrm{OC}_{2} \mathrm{H}_{5}$ & $-\mathrm{SO}_{2}-\mathrm{C}_{6} \mathrm{H}_{4}-4-\mathrm{CH}_{3}$ & $\begin{array}{l}\mathrm{C}_{27} \mathrm{H}_{28} \mathrm{~N}_{4} \mathrm{O}_{3} \mathrm{~S} \\
(488)\end{array}$ & $213-215$ & 61.1 & $\begin{array}{l}66.39 \\
66.20 \\
\end{array}$ & $\begin{array}{l}5.73 \\
5.54 \\
\end{array}$ & $\begin{array}{l}11.47 \\
11.62 \\
\end{array}$ \\
\hline $6 \mathrm{a}$ & $\mathrm{H}$ & $-\mathrm{SO}_{2}-\mathrm{C}_{6} \mathrm{H}_{4}-\mathrm{NH}-\mathrm{CO}-\mathrm{CH}_{3}$ & $\begin{array}{l}\mathrm{C}_{26} \mathrm{H}_{25} \mathrm{~N}_{5} \mathrm{O}_{3} \mathrm{~S} \\
(487)\end{array}$ & $158-161$ & 76.3 & $\begin{array}{l}64.04 \\
64.12 \\
\end{array}$ & $\begin{array}{l}5.13 \\
4.95 \\
\end{array}$ & $\begin{array}{l}14.37 \\
14.26 \\
\end{array}$ \\
\hline $6 \mathrm{~b}$ & $4-\mathrm{Cl}$ & $-\mathrm{SO}_{2}-\mathrm{C}_{6} \mathrm{H}_{4}-\mathrm{NH}-\mathrm{CO}-\mathrm{CH}_{3}$ & $\begin{array}{l}\mathrm{C}_{26} \mathrm{H}_{25} \mathrm{~N}_{5} \mathrm{O}_{3} \mathrm{~S} \\
(521)\end{array}$ & $123-125$ & 72.2 & $\begin{array}{l}59.88 \\
60.11 \\
\end{array}$ & $\begin{array}{l}4.60 \\
4.55 \\
\end{array}$ & \begin{tabular}{|l|}
13.43 \\
13.20 \\
\end{tabular} \\
\hline $6 c$ & $4-\mathrm{OC}_{2} \mathrm{H}_{5}$ & $-\mathrm{SO}_{2}-\mathrm{C}_{6} \mathrm{H}_{4}-\mathrm{NH}-\mathrm{CO}-\mathrm{CH}_{3}$ & $\begin{array}{l}\mathrm{C}_{28} \mathrm{H}_{29} \mathrm{~N}_{5} \mathrm{O}_{4} \mathrm{~S} \\
(531)\end{array}$ & $189-191$ & 68.7 & $\begin{array}{l}63.27 \\
63.35 \\
\end{array}$ & $\begin{array}{l}5.46 \\
5.52 \\
\end{array}$ & \begin{tabular}{|l|}
13.18 \\
13.24 \\
\end{tabular} \\
\hline $6 d$ & $4-\mathrm{OH}$ & $-\mathrm{SO}_{2}-\mathrm{C}_{6} \mathrm{H}_{4}-\mathrm{NH}-\mathrm{CO}-\mathrm{CH}_{3}$ & $\begin{array}{l}\mathrm{C}_{26} \mathrm{H}_{25} \mathrm{~N}_{5} \mathrm{O}_{4} \mathrm{~S} \\
(503)\end{array}$ & $160-162$ & 75.2 & $\begin{array}{l}62.02 \\
62.25 \\
\end{array}$ & $\begin{array}{l}4.97 \\
4.85 \\
\end{array}$ & \begin{tabular}{|l|}
13.91 \\
13.82 \\
\end{tabular} \\
\hline $6 e$ & $4-\mathrm{CH}_{3}$ & $-\mathrm{SO}_{2}-\mathrm{C}_{6} \mathrm{H}_{4}-\mathrm{NH}-\mathrm{CO}-\mathrm{CH}_{3}$ & $\begin{array}{l}\mathrm{C}_{27} \mathrm{H}_{27} \mathrm{~N}_{5} \mathrm{O}_{3} \mathrm{~S} \\
(501)\end{array}$ & $150-152$ & 72.4 & $\begin{array}{l}64.67 \\
64.73 \\
\end{array}$ & $\begin{array}{l}5.38 \\
5.42 \\
\end{array}$ & \begin{tabular}{|l|}
13.97 \\
13.99 \\
\end{tabular} \\
\hline $7 a$ & $\mathrm{H}$ & $-\mathrm{SO}_{2}-\mathrm{C}_{6} \mathrm{H}_{4}-\mathrm{NH}_{2}$ & $\begin{array}{l}\mathrm{C}_{24} \mathrm{H}_{23} \mathrm{~N}_{5} \mathrm{O}_{2} \mathrm{~S} \\
(445)\end{array}$ & $110-112$ & 84.4 & $\begin{array}{l}69.73 \\
70.12 \\
\end{array}$ & $\begin{array}{l}5.56 \\
5.63 \\
\end{array}$ & \begin{tabular}{|l|}
16.94 \\
17.25 \\
\end{tabular} \\
\hline $7 \mathrm{~b}$ & $4-\mathrm{Cl}$ & $-\mathrm{SO}_{2}-\mathrm{C}_{6} \mathrm{H}_{4}-\mathrm{NH}_{2}$ & $\begin{array}{l}\mathrm{C}_{24} \mathrm{H}_{22} \mathrm{~N}_{5} \mathrm{O}_{2} \mathrm{SCl} \\
(479)\end{array}$ & $174-176$ & 81.7 & $\begin{array}{l}60.12 \\
60.23 \\
\end{array}$ & $\begin{array}{l}4.59 \\
4.70 \\
\end{array}$ & $\begin{array}{l}14.61 \\
14.72 \\
\end{array}$ \\
\hline $7 \mathrm{c}$ & $4-\mathrm{OC}_{2} \mathrm{H}_{5}$ & $-\mathrm{SO}_{2}-\mathrm{C}_{6} \mathrm{H}_{4}-\mathrm{NH}_{2}$ & $\begin{array}{l}\mathrm{C}_{26} \mathrm{H}_{2} \mathrm{~N}_{5} \mathrm{O}_{3} \mathrm{~S} \\
(4899)\end{array}$ & $178-180$ & 77.2 & $\begin{array}{l}63.80 \\
63.55 \\
\end{array}$ & \begin{tabular}{|l|}
5.52 \\
5.45 \\
\end{tabular} & \begin{tabular}{|l|}
14.31 \\
14.48 \\
\end{tabular} \\
\hline $7 \mathrm{~d}$ & $4-\mathrm{OH}$ & $-\mathrm{SO}_{2}-\mathrm{C}_{6} \mathrm{H}_{4}-\mathrm{NH}_{2}$ & $\begin{array}{l}\mathrm{C}_{24} \mathrm{H}_{23} \mathrm{~N}_{5} \mathrm{O}_{3} \mathrm{~S} \\
(461)\end{array}$ & $160-162$ & 75.3 & $\begin{array}{l}62.47 \\
62.55 \\
\end{array}$ & \begin{tabular}{|l|}
4.98 \\
5.12 \\
\end{tabular} & \begin{tabular}{|l|}
15.18 \\
15.20 \\
\end{tabular} \\
\hline $7 e$ & $4-\mathrm{CH}_{3}$ & $-\mathrm{SO}_{2}-\mathrm{C}_{6} \mathrm{H}_{4}-\mathrm{NH}_{2}$ & $\begin{array}{l}\mathrm{C}_{25} \mathrm{H}_{25} \mathrm{~N}_{5} \mathrm{O}_{2} \mathrm{~S} \\
(459)\end{array}$ & $125-127$ & 72.2 & $\begin{array}{l}65.35 \\
65.40\end{array}$ & $\begin{array}{l}5.44 \\
5.25\end{array}$ & $\begin{array}{l}15.25 \\
15.33\end{array}$ \\
\hline
\end{tabular}

Table 1 
The obtained chemical compounds were examined from the point of view of their microbiological activity, which was assessed on selected bacterial strains.

Assessment of the activity of ivestigated substances was carried out according to FP V [8]. Absolute activity was determined by defining lowest concentration able to inhibit the growth of bacterial stain (MIC) in fluid medium and wxpressed in micrograms $(\mu \mathrm{g})$ of investigated substanced per $1 \mathrm{ml}$. Relative activity was determined MIC value of the investigated substance by MIC value of a pattern (Urenil, Erytromycin).

Antibacterial potential was determined by means of cylinder-plate method assuming the average from 9 trials ( 3 weighed samples by 3 measurements).

The obtained results are presented in Table 2, 3 .

Table 2: Antibacterial activity (MIC, $\mu \mathrm{g} / \mathrm{ml})$

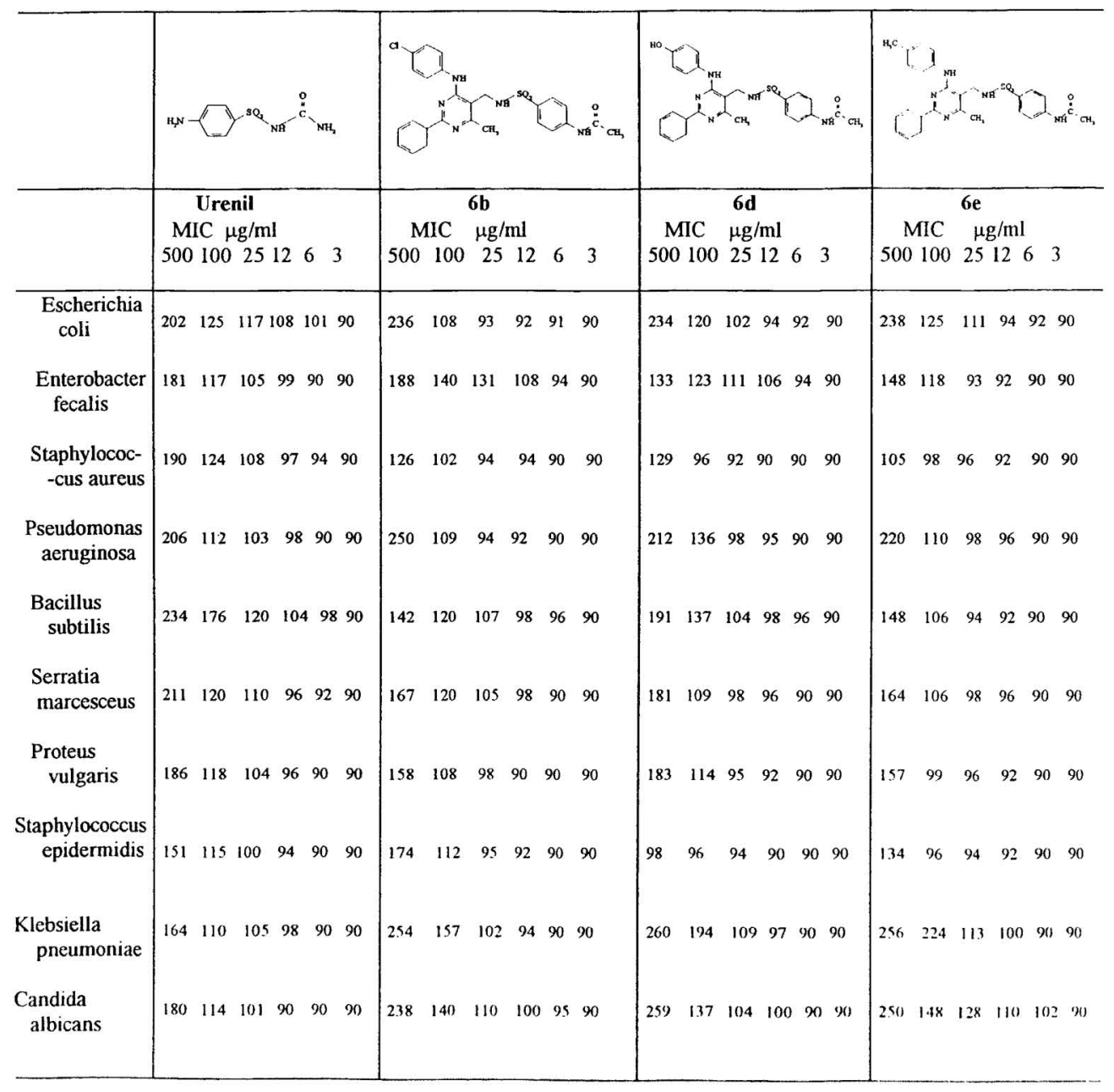


Table 3: Antibacterial activity (MIC, $\mu \mathrm{g} / \mathrm{ml})$

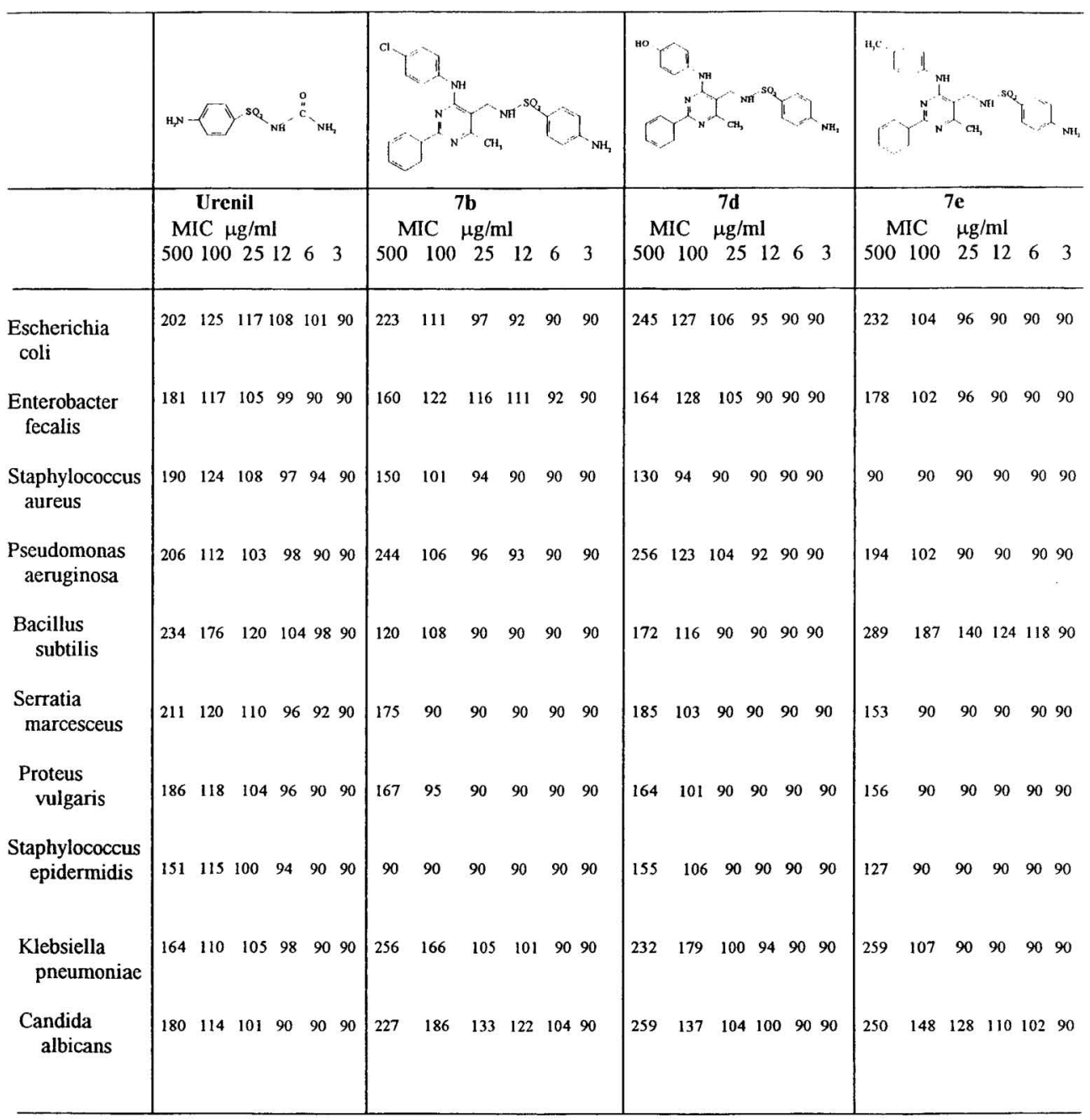

\section{RESULTS AND DISCUSSION}

Seven newly obtained pyrimidine derivatives were studied microbiologically on 10 bacterial strains Fscherichia coli, Enterococcus faecalis, Staphylococcus aureus, Pseudomonas aeruginosa, Bacillus subtilis, Serratia marcesceus, Proteus vilgaris, Staphylococcus epidermidis, Klebsiella pneomonae, 
Candida albicans. In comparison with the pattern (sulphonamide used in medicine - Urenil), the obtained compounds have revealed interesting antibacterial as well as antifungal activity. Compounds 6b, 6d, 6e have revealed satisfactory antibacterial properties, however their activity increased significantly when the amine group in phenyl ring of sulphonamide group was deblocked from acetyl radical (compounds $\mathbf{7 b}, \mathbf{7 d}, \mathbf{7 e}$ ). The activity decreased significantly if alkyl radical was used to replace the amino group in phenyl ring of sulphonamide group as in compound 4 . Phenylamine substituent at site 4 of the pyrimidine ring also plays an important role. Substitution of p-chloroaniline, p-aminophenol or p-toluidine at carbon 4 of the pyrimidine ring increases significantly the antibacterial activity. The above mentioned derivatives reveal extremely potent properties of inhibiting the growth of Gram (+) Enterobacter faecalis, Gram (-) Pseudomonas aeruginosa, Gram (-) Klebsiella pneumoniae.

It should be stressed that above-mentioned sulphoderivatives 7 also reveal antifungal properties, inhibiting strongly the growth of Candida albicans, what holds promise in further research.

\section{Expeimental}

Melting points were determined in Köfler apparatus.

${ }^{1} \mathrm{H}$ NMR spectra were recorded on BS-487-C, $80 \mathrm{MHz}$ Tesla spectrometer. Infrared (IR) spectra were recorded in nujol with a Specord spectrophotometer at Analytical Laboratory of Medical Academy in Wrocław. Elemental analyses indicated by the symbols were within $+1-0.4 \%$ of the theoretical values.

\section{6-Methyl-2-phenyl-4-phenylamine-5-aminomethylpyrimidine (3a)}

$4 \mathrm{~g}$ (12.9 mmol) of 6-methyl-2-phenyl-4-phenylamine-5-chloromethyl-pyrimidine (2a $)$ were added to $30 \mathrm{ml}$ of $25 \%$ aqueous solution of ammonia. The mixture was heated under reflux condenser and stirred intensively throughout the process. After $5 \mathrm{~h}$ the postreaction mixture was diluted with $100 \mathrm{ml}$ of water and three times extracted with chloroform. The chloroform extracts were combined, dried over $\mathrm{MgSO}_{4}$ and after filtration vacuum condensed. Obtained oily product was crystallised from acetone - chloroform 1:1 mixture, giving $3.46 \mathrm{~g}(92.3 \%)$ of cream-white crystals with m.p. $117-119^{\circ} \mathrm{C}$.

Spectra data of 3a IR $\left[v, \mathrm{~cm}^{-1}\right]: 2924(\mathrm{NH}), 1442(\mathrm{NH}), 1378\left(\mathrm{NH}_{2}\right)$.

${ }^{1} \mathrm{H}$ NMR [ $\left.\delta, \mathrm{ppm}, \mathrm{CDCl}_{3}\right]: 2.45\left(\mathrm{~s}, 3 \mathrm{H} ; \mathrm{CH}_{3}\right), 4.30\left(\mathrm{t}, 2 \mathrm{H} ; \mathrm{CH}_{2}\right), 5.12(\mathrm{~s}, 1 \mathrm{H} ; \mathrm{NH}) 6.80-8.45(\mathrm{~m}, 10 \mathrm{H}$ aromatic), $9.58(\mathrm{~s}, 1 \mathrm{H} ; \mathrm{ArNH})$.

Spectral data of 3b: IR [ $\mathrm{v}, \mathrm{cm}^{-1}$ ]]: $2924(\mathrm{NH}), 1554(\mathrm{NH}), 1490\left(\mathrm{NH}_{2}\right)$

${ }^{1} \mathrm{H} \mathrm{NMR}\left[\delta, \mathrm{ppm}, \mathrm{CDCl}_{3}\right]: 2.50\left(\mathrm{~s}, 3 \mathrm{H} ; \mathrm{CH}_{3}\right), 4.35\left(\mathrm{t}, 2 \mathrm{H} ; \mathrm{CH}_{2}\right), 5.26\left(\mathrm{t}, 2 \mathrm{H} ; \mathrm{NH}_{2}\right), 6.70-8.55(\mathrm{~m}$, $9 \mathrm{H}$ aromatic), 9.12 (s, $1 \mathrm{H} ; \mathrm{ArNH})$.

Spectral data of 3c: IR $\left[v, \mathrm{~cm}^{-1}\right]: 2930(\mathrm{NH}), 1455(\mathrm{NH}), 1395\left(\mathrm{NH}_{2}\right)$.

${ }^{1} \mathrm{H}$ NMR [ $\delta$, ppm, $\left.\mathrm{CDCl}_{3}\right]: 1.25\left(\mathrm{t}, 3 \mathrm{H} ; \mathrm{CH}_{3}\right), 2.60\left(\mathrm{~s}, 3 \mathrm{H} ; \mathrm{CH}_{3}\right), 3.70\left(\mathrm{q}, 2 \mathrm{H} ; \mathrm{CH}_{2}\right), 4.40(\mathrm{t}, 2 \mathrm{H}$; $\left.\mathrm{CH}_{2}\right), 4.70(\mathrm{~s}, 1 \mathrm{H} ; \mathrm{ArNH}), 5.30\left(\mathrm{t}, 2 \mathrm{H} ; \mathrm{NH}_{2}\right), 7.20-850(\mathrm{~m}, 9 \mathrm{H} ;$ aromatic), $9.20(\mathrm{~s}, 1 \mathrm{H} ; \mathrm{ArNH})$.

Spectral data of 3d: IR [ $\left.\mathrm{v}, \mathrm{cm}^{-1}\right]: 2930(\mathrm{NH}), 1550(\mathrm{NH}), 1495\left(\mathrm{NH}_{2}\right)$.

${ }^{1} \mathrm{H}$ NMR [ $\delta$, ppm, $\left.\mathrm{CDCl}_{3}\right]: 2.55\left(\mathrm{~s}, 3 \mathrm{H} ; \mathrm{CH}_{3}\right), 4.30\left(\mathrm{t}, 2 \mathrm{H} ; \mathrm{CH}_{2}\right), 5.30\left(\mathrm{t}, 2 \mathrm{H} ; \mathrm{NH}_{2}\right), 5.58(\mathrm{~s}, 1 \mathrm{H} ; \mathrm{Ar}-$ $\mathrm{OH}), 6.74-8.55(\mathrm{~m}, 9 \mathrm{H}$ aromatic), $9.15(\mathrm{~s}, 1 \mathrm{H} ; \mathrm{ArNH})$. 
Spectral data of 3e: IR $\left[\mathrm{V}, \mathrm{cm}^{-1}\right]: 2940(\mathrm{NH}), 1560(\mathrm{NH}), 1498\left(\mathrm{NH}_{2}\right)$.

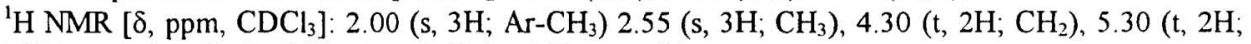
$\left.\mathrm{NH}_{2}\right), 5.58(\mathrm{~s}, 1 \mathrm{H} ; \mathrm{Ar}-\mathrm{OH}), 6.74-8.55(\mathrm{~m}, 9 \mathrm{H}$ aromatic $)$,

$9.15(\mathrm{~s}, 1 \mathrm{H} ; \mathrm{ArNH})$.

6-Methyl-4-(phenylamine)-2-phenyl-5-(4'-methylphenyl)sulphonamidomethyl-pyrimidine (4b)

$4 \mathrm{~g}(13.7 \mathrm{mmol})$ of 6-methyl-4-phenylamine-2-phenyl-5-aminmethylpyrimidine (3a) were diluted in $50 \mathrm{ml}$ of benzene and $2.5 \mathrm{~g}$ of p-toluensulphonic chloride were added gradually. The mixture was heated under reflux condenser for $8 \mathrm{~h}$. Next the postreaction mixture was cooled and poured to $100 \mathrm{ml}$ of water. The solution was three times extracted with $50 \mathrm{ml}$ of chloroform. The chloroform extracts were combined and dried over $\mathrm{MgSO}_{4}$, and next after filtration vacuum condensed. Oily residue was purified on chromatographic column filled with silica gel 60 (35-70 mesh ASTM), using the mixture of chloroform-acetone 3:1. $3.9 \mathrm{~g}(64.5 \%)$ of sulphonamide $4 \mathrm{a}$ with m.p. of $164-166^{\circ} \mathrm{C}$ were obtained.

Spectra data of 4a IR $\left[\mathrm{v}, \mathrm{cm}^{-1}\right]: 2040(\mathrm{NH}), 1450(\mathrm{NH}), 1308\left(\mathrm{SO}_{2}\right)$.

${ }^{1} \mathrm{H}$ NMR $\left[\delta, \mathrm{ppm}, \mathrm{CDCl}_{3}\right]: 2.55\left(\mathrm{~s} .3 \mathrm{H} ; \mathrm{CH}_{3}\right), 3.45$ (s. $\left.3 \mathrm{H} ; \mathrm{CH}_{3}\right), 6.25(1 \mathrm{H} ; \mathrm{NH}), \quad 7.25-8.60(14 \mathrm{H}$; aromatic).

Spectral data of 4b: IR [ $\left.\mathrm{v}, \mathrm{cm}^{-1}\right]: 2940(\mathrm{NH}), 1560(\mathrm{NH}), 1498\left(\mathrm{NH}_{2}\right)$.

${ }^{1} \mathrm{H}$ NMR $\left[\delta, \mathrm{ppm}, \mathrm{CDCl}_{3}\right.$ ]: $2.50\left(\mathrm{~s}, 3 \mathrm{H} ; \mathrm{CH}_{3}\right), 3.40\left(\mathrm{~s}, 3 \mathrm{H} ; \mathrm{CH}_{3}\right), 4.40\left(\mathrm{~d}, 2 \mathrm{H} ; \mathrm{CH}_{2}\right), 4.75(\mathrm{~s}, 1 \mathrm{H}$; $\operatorname{ArNH}), 6.25(\mathrm{t}, 1 \mathrm{H} ; \mathrm{NH})$.

Spectral data of 4c: IR $\left[v, \mathrm{~cm}^{-1}\right]: 2950(\mathrm{NH}), 1415(\mathrm{NH}), 1312\left(\mathrm{SO}_{2}\right)$.

${ }^{1} \mathrm{H}$ NMR [ $\delta, \mathrm{ppm}, \mathrm{CDCl}_{3}$ ]: $1.70\left(\mathrm{t}, 3 \mathrm{H} ; \mathrm{CH}_{3}\right), 2.45\left(\mathrm{~s}, 3 \mathrm{H} ; \mathrm{CH}_{3}\right), 3.30\left(\mathrm{~s}, 3 \mathrm{H} ; \mathrm{CH}_{3}\right), 3.80(\mathrm{q}, 2 \mathrm{H}$; $\left.\mathrm{CH}_{2}\right), 4.35\left(\mathrm{~d}, 2 \mathrm{H} ; \mathrm{CH}_{2}\right), 4.70(\mathrm{~s}, 1 \mathrm{H} ; \mathrm{ArNH}), 6.20(\mathrm{t}, 1 \mathrm{H} ; \mathrm{NH}), 7.30-8.60(\mathrm{~m}, 13 \mathrm{H}$; aromatic)

\section{6-Methyl-2-phenyl-4-phenylamine-5-(4'-N-acetylaminphenyl)-sulphonamido- methylpyrimidine (6a)}

$4 \mathrm{~g}(12.9 \mathrm{mmol})$ of 6-methyl-2-phenyl-6-phenylamin-5-chloromethylpyrimidine (2) were diluted in $50 \mathrm{ml}$ of chloroform and $3 \mathrm{~g}$ of $\mathrm{N}$-acetylsulphanilic acid amide were added. The mixture was heated for $12 \mathrm{~h}$ under reflux condenser and stirred vigorously. Next, the mixture was cooled and $100 \mathrm{ml}$ of water was added. The mixture was extracted three times with $50 \mathrm{ml}$ of chloroform. The chloroform extracts were combined, dried over $\mathrm{MgSO}_{4}$ and after filtration vacuum condensed. Oily residue was purified chromatographically with silica gel 60 (35-70 mesh ASTM) using chloroform-aceton $3: 1$ mixture, giving $4.8 \mathrm{~g}(76.3 \%)$ of precipitate with m.p. $158-161^{\circ} \mathrm{C}$.

Spectra data of $\operatorname{IR}\left[\mathrm{v}, \mathrm{cm}^{-1}\right]: 2836(\mathrm{NH}), 1556(\mathrm{NH}), 1496\left(\mathrm{SO}_{2}\right)$.

${ }^{1} \mathrm{H}$ NMR $\left[\delta, \mathrm{ppm}, \mathrm{CDCl}_{3}\right]: 1.30(\mathrm{~s}, \mathrm{lH} ; \mathrm{AcNH}), 2.55\left(\mathrm{~s}, 3 \mathrm{H} ; \mathrm{CH}_{3}\right), 3.50\left(\mathrm{~s}, 3 \mathrm{H} ; \mathrm{CH}_{3}\right), 4.75(\mathrm{~d}, 2 \mathrm{H}$; $\left.\mathrm{CH}_{2}\right), 4.80(\mathrm{~s}, 1 \mathrm{H} ; \mathrm{ArNH}), 5.20(\mathrm{t}, 1 \mathrm{H} ; \mathrm{NH}), 7.35-8.55$ (m, 14H;aromatic).

Spectral data of 6b: IR [ $\left.\mathrm{v}, \mathrm{cm}^{-1}\right]: 2940(\mathrm{NH}), 1560(\mathrm{NH}), 1498\left(\mathrm{NH}_{2}\right)$.

${ }^{1} \mathrm{H}$ NMR [ $\left.\delta, \mathrm{ppm}, \mathrm{CDCl}_{3}\right]: 1.25(\mathrm{~s}, 1 \mathrm{H} ; \mathrm{AcNH}), 2.50\left(\mathrm{~s}, 3 \mathrm{H} ; \mathrm{CH}_{3}\right), 3.45\left(\mathrm{~s}, 3 \mathrm{H} ; \mathrm{CH}_{3}\right), 4.70(\mathrm{~d}, 2 \mathrm{H}$; $\left.\mathrm{CH}_{2}\right), 4.75(\mathrm{~s}, 1 \mathrm{H} ; \mathrm{ArNH}), 5.20(\mathrm{~d}, 1 \mathrm{H} ; \mathrm{NH}), 7.30-8.50(\mathrm{~m}, 13 \mathrm{H}$; aromatic)

Spectral data of 6c: IR $\left[\mathrm{v}, \mathrm{cm}^{-1}\right]: 2940(\mathrm{NH}), 1560(\mathrm{NH}), 1498\left(\mathrm{NH}_{2}\right)$. 
${ }^{1} \mathrm{H}$ NMR [ $\left.\delta, \mathrm{ppm}, \mathrm{CDCl}_{3}\right]: 1.20(\mathrm{~s}, 1 \mathrm{H} ; \mathrm{AcNH}), 1.30\left(\mathrm{t}, 3 \mathrm{H} ; \mathrm{CH}_{3}\right), 2.20\left(\mathrm{~s}, 3 \mathrm{H} ; \mathrm{CH}_{3}\right), 2.35(\mathrm{~s}, 3 \mathrm{H}$; $\left.\mathrm{CH}_{3}\right), 3.40$ (q, $\left.2 \mathrm{H} ; \mathrm{CH}_{2}\right), 3.60(\mathrm{t}, 1 \mathrm{H} ; \mathrm{NH}), 3.85\left(\mathrm{~d}, 2 \mathrm{H} ; \mathrm{CH}_{2}\right), 4.70(\mathrm{~s}, 1 \mathrm{H} ; \mathrm{ArNH})$

Spectral data of 6d: IR [ $\left.\mathrm{v}, \mathrm{cm}^{-1}\right]: 2840(\mathrm{NH}), 1560(\mathrm{NH}), 1490\left(\mathrm{SO}_{2}\right)$.

${ }^{1} \mathrm{H}$ NMR [ 8 , ppm. $\left.\mathrm{CDCl}_{3}\right]: 1.40(\mathrm{~s}, 1 \mathrm{H} ; \mathrm{AcNH}), 2.60\left(\mathrm{~s}, 3 \mathrm{H} ; \mathrm{CH}_{3}\right), 3.55\left(\mathrm{~s}, 3 \mathrm{H} ; \mathrm{CH}_{3}\right), 4.85(\mathrm{~d}, 2 \mathrm{H}$; $\left.\mathrm{CH}_{2}\right), 4.80(\mathrm{~s}, 1 \mathrm{H} ; \mathrm{ArNH}), 5.20(\mathrm{t}, 1 \mathrm{H} ; \mathrm{NH}), 5.50(\mathrm{~s}, 1 \mathrm{H} ; \mathrm{Ar}-\mathrm{OH}), 7.35-8.55(\mathrm{~m}, 14 \mathrm{H}$; aromatic $)$

Spectral data of 6e: IR $\left[v, \mathrm{~cm}^{-1}\right]: 2850(\mathrm{NH}), 1550(\mathrm{NH}), 1495\left(\mathrm{SO}_{2}\right)$

${ }^{1} \mathrm{H}$ NMR [ $\delta, \mathrm{ppm}, \mathrm{CDCl}_{3}$ ]: 1.40(s, $\left.1 \mathrm{H} ; \mathrm{AcNH}\right), 2.20\left(\mathrm{~s}, 3 \mathrm{H} ; \mathrm{Ar}-\mathrm{CH}_{3}\right), 2.60\left(\mathrm{~s}, 3 \mathrm{H} ; \mathrm{CH}_{3}\right), 3.55(\mathrm{~s}, 3 \mathrm{H}$; $\left.\mathrm{CH}_{3}\right), 4.75\left(\mathrm{~d}, 2 \mathrm{H} ; \mathrm{CH}_{2}\right), 4.70(\mathrm{~s}, 1 \mathrm{H} ; \mathrm{ArNH}), 5.30(\mathrm{t}, 1 \mathrm{H} ; \mathrm{NH}), 5.50(\mathrm{~s}, 1 \mathrm{H} ; \mathrm{Ar}-\mathrm{OH}), 7.35-8.55(\mathrm{~m}$, $14 \mathrm{H}$; aromatic).

\section{6-Methyl-2-phenyl-4-phenylamine-5(4'-aminphenyl)-sulphonamidomethyl-pyrimidine (7a)}

$4 \mathrm{~g}(8.2 \mathrm{mmol})$ of 6-methyl-2-phenyl-4-phenylamine-5-(4'-acetylphenyl)-sulphonamidomethyl-pyrimidine (6a) were diluted in $50 \mathrm{ml}$ of alcoholic solution of $\mathrm{HCl}$. The mixture was heated under reflux condenser for $1 \mathrm{~h}$. Next the solution was cooled, $100 \mathrm{ml}$ of water were added and three times extracted with $50 \mathrm{ml}$ of chloroform. The chloroform extracts were combined, dried over $\mathrm{MgSO}_{4}$ and after filtration vacuum condensed. Oily residue was purified on chloroform-acetone $3: 1 \mathrm{column}$, giving crystals with m.p. $110-112^{\circ} \mathrm{C}$.

Spectra data of $\mathbb{R}\left[\mathrm{v}, \mathrm{cm}^{-1}\right.$, nujol]: $2836(\mathrm{NH}), 1562\left(\mathrm{NH}_{2}\right), 1492(\mathrm{NH}), 1406\left(\mathrm{SO}_{2}\right)$. 'H NMR [80-MHz, $\delta, p p m, \mathrm{CDCl}_{3}$ ]: 1.25 (s. $2 \mathrm{H} ; \mathrm{NH}_{2}$ ), 2.45 (s. $\left.3 \mathrm{H} ; \mathrm{CH}_{3}\right), 3.25(\mathrm{t} .1 \mathrm{H} ; \mathrm{NH}), 4.50$ (d. $2 \mathrm{H} ; \mathrm{CH}_{2}$ ), 4.75 (s. $\left.1 \mathrm{H} ; \mathrm{ArNH}\right), 7.40-8.50$ (m. 14H; aromatic).

Spectral data of 7b: IR [v, cm $\left.{ }^{-1}\right]: 2885(\mathrm{NH}), 16775\left(\mathrm{NH}_{2}\right), 1455(\mathrm{NH}), 1410\left(\mathrm{SO}_{2}\right)$ ${ }^{1} \mathrm{H}$ NMR $\left[\delta, \mathrm{ppm}, \mathrm{CDCl}_{3}\right]: 1.30\left(\mathrm{~s}, 2 \mathrm{H} ; \mathrm{NH}_{2}\right), 2.50\left(\mathrm{~s}, 3 \mathrm{H} ; \mathrm{CH}_{3}\right), 3.30(\mathrm{t}, 1 \mathrm{H} ; \mathrm{NH}), 4.45\left(\mathrm{~d}, 2 \mathrm{H} ; \mathrm{CH}_{2}\right)$ $4.70(\mathrm{~s}, 1 \mathrm{H} ; \mathrm{NH}), 7.40-8.50(\mathrm{~m}, 13 \mathrm{H}$; aromatic).

Spectral data of 7c: IR [ $\left.\mathrm{v}, \mathrm{cm}^{-1}\right]: 2850(\mathrm{NH}), 1660\left(\mathrm{NH}_{2}\right), 1460(\mathrm{NH}), 1407\left(\mathrm{SO}_{2}\right)$ ${ }^{1} \mathrm{H}$ NMR [ $\delta$, ppm, $\left.\mathrm{CDCl}_{3}\right]: 1.20\left(\mathrm{~s}, 2 \mathrm{H} ; \mathrm{NH}_{2}\right), 1.35\left(\mathrm{t}, 3 \mathrm{H} ; \mathrm{CH}_{3}\right), 2.45\left(\mathrm{~s}, 3 \mathrm{H} ; \mathrm{CH}_{3}\right), 3.20\left(\mathrm{q}, 2 \mathrm{H} ; \mathrm{CH}_{2}\right.$, $3.50(\mathrm{t}, 1 \mathrm{H} ; \mathrm{NH}), 3.75\left(\mathrm{~d}, 2 \mathrm{H} ; \mathrm{CH}_{2}\right), 3.80(\mathrm{~s}, 1 \mathrm{H} ; \mathrm{ArNH}), 7.45-8.55(\mathrm{~m}, 13 \mathrm{H}$; aromatic)

Spectral data of 7d: IR $\left[\mathrm{v}, \mathrm{cm}^{-1}\right]: 2895(\mathrm{NH}), 1670\left(\mathrm{NH}_{2}\right), 1445(\mathrm{NH}), 1415\left(\mathrm{SO}_{2}\right)$

${ }^{1} \mathrm{H}$ NMR [ $\delta$, ppm, $\mathrm{CDCl}_{3}$ ]: $1.30\left(\mathrm{~s}, 2 \mathrm{H} ; \mathrm{NH}_{2}\right), 2.50\left(\mathrm{~s}, 3 \mathrm{H} ; \mathrm{CH}_{3}\right), 3.25(\mathrm{t}, \mathrm{lH} ; \mathrm{NH}), 4.40\left(\mathrm{~d}, 2 \mathrm{H} ; \mathrm{CH}_{2}\right)$, $4.70(\mathrm{~s}, 1 \mathrm{H} ; \mathrm{NH}), 5.50(\mathrm{~s}, 1 \mathrm{H} ; \mathrm{Ar}-\mathrm{OH}), 7.40-8.50(\mathrm{~m}, 13 \mathrm{H}$; aromatic).

Spectral data of 7e: IR [ $\left.v, \mathrm{~cm}^{-1}\right]: 2875(\mathrm{NH}), 1660\left(\mathrm{NH}_{2}\right), 1455(\mathrm{NH}), 1425\left(\mathrm{SO}_{2}\right)$

${ }^{1} \mathrm{H}$ NMR [ $\left.\delta, \mathrm{ppm}, \mathrm{CDCl}_{3}\right]: 1.40\left(\mathrm{~s}, 2 \mathrm{H} ; \mathrm{NH}_{2}\right), 2.20\left(\mathrm{~s}, 3 \mathrm{H} ; \mathrm{Ar}-\mathrm{CH}_{3}\right), 2.60\left(\mathrm{~s}, 3 \mathrm{H}-\mathrm{CH}_{3}\right), 3.35(\mathrm{t}, 1 \mathrm{H}$; $\mathrm{NH}), 4.40\left(\mathrm{~d}, 2 \mathrm{H} ; \mathrm{CH}_{2}\right), 4.70(\mathrm{~s}, 1 \mathrm{H} ; \mathrm{NH}), 5.50(\mathrm{~s}, 1 \mathrm{H} ; \mathrm{Ar}-\mathrm{OH}), 7.40-8.50(\mathrm{~m}, 13 \mathrm{H}$; aromatic)

\section{REFERENCES}

[1] Z. Machoń, J. Cieplik, Synthesis 2, 142, (1986).

[2] Z. Machoń, J. Cieplik, Pol. J. Pharmacol. Pharm. 40, 201, (1988)

[3] J. Cieplik, Z. Machon, M. Zimecki, Z. Wieczorek, Arch. Imm. -Ther. Exp. 4 , 11, (1993). 
[4] J. Cieplik, Z. Machó́, M. Zimecki, Z. Wieczorek, Il Farmaco 50, 131, (1995)

[5] Z. Machoń, J. Cieplik, Eur. J. Chem-Chim. Ther. 19, (4), 113, (1994).

[6] J. Pluta, M. Flendrich, J. Cieplik, J. Boll. Chim. Farmaceutico-Anno135, 239, (1996).

[7] J. Cieplik, J. Pluta, A. Meler, Arch. Pharm. Med. Chem., 333, 237, (1997).

[8] Pharmacopea polonica V (1990), I, 94. 